\title{
Epidemiology and Virulence Determinants including Biofilm Profile of Candida Infections in an ICU in a Tertiary Hospital in India
}

\author{
Ravinder Kaur, ${ }^{1}$ Ritu Goyal, ${ }^{1}$ Megh S. Dhakad, ${ }^{1}$ Preena Bhalla, ${ }^{1}$ and Rakesh Kumar ${ }^{2}$ \\ ${ }^{1}$ Department of Microbiology, Maulana Azad Medical College \& Associated Lok Nayak Hospitals, New Delhi 110002, India \\ ${ }^{2}$ Department of Anesthesiology, Maulana Azad Medical College \& Associated Lok Nayak Hospitals, New Delhi 110002, India
}

Correspondence should be addressed to Ravinder Kaur; rkaur.mamc@gmail.com

Received 12 October 2013; Revised 5 December 2013; Accepted 5 December 2013; Published 12 January 2014

Academic Editor: Praveen Rao Juvvadi

Copyright (C) 2014 Ravinder Kaur et al. This is an open access article distributed under the Creative Commons Attribution License, which permits unrestricted use, distribution, and reproduction in any medium, provided the original work is properly cited.

\begin{abstract}
The purpose of this prospective study was to isolate, speciate, and determine antifungal susceptibility and virulence patterns of Candida species recovered from the intensive care units (ICUs) in an Indian hospital. Study included 125 medical/postoperative patients admitted to ICU. Identification and speciation of yeast isolates were done by the biochemical methods. Antifungal susceptibility was done by broth microdilution method. Virulence testing of Candida species was done by phospholipase, proteinase, and adherence assay. A total of 103 Candida isolates were isolated; C. tropicalis was the predominant species (40.7\%), followed by C. albicans (38.83 \%), C. glabrata (11.65\%), C. parapsilosis (3.88\%), and $1.94 \%$ each of C. krusei, C. kefyr, and C. sphaerica. 60 Candida isolates (58.25\%) showed resistance to fluconazole, while 7 (6.7\%) isolates showed resistance to amphotericin B. Phospholipase and proteinase activities were seen in $73.8 \%$ and $55.3 \%$ Candida isolates with different species showing a wide range of activities, while $68.9 \%$ Candida isolates showed $4+$ adherence activity. The present study revealed that nonalbicans Candida species (NAC spp.) caused most of the cases of Candidemia in the ICU patients. The isolation of C. tropicalis from a large number of cases highlights the ability of this pathogen to cause bloodstream infections. The presence of azole resistance is a matter of concern.
\end{abstract}

\section{Introduction}

Fungal infections are emerging as a problem of significant magnitude in hospitals during the last decade, especially in the ICUs, which are epicenters for infections such as Candidemia and invasive Candida infection (ICI). The escalating problem in ICUs is probably due to an increasing population of immunocompromised patients. A survey of the epidemiology of sepsis conducted in USA revealed that the incidence of fungal sepsis increased threefold between 1979 and 2000 [1]. Fungal infections account for nearly 8\% of all nosocomial infections; Candida is the responsible agent in $80 \%$ of the cases [2]. Candida species are approximately the fourth most common cause of nosocomial infections in ICUs, according to data from the National Nosocomial
Infections Surveillance System and the European Prevalence of Infection in Intensive Care [3].

Critically ill patients who are treated in the ICUs are very susceptible to infections due to acquired defects in host defense mechanisms from the immunosuppressive effect of the underlying disease, recent surgery, trauma, and concurrent drug therapy. Infections occur in $15-40 \%$ of all ICU admissions and central venous catheters are considered as the most common risk factor for the development of Candidemia in these patients $[4,5]$. The Candida biofilm results in antifungal drug resistance and protection of the fungus from host defenses [6], which carry important clinical repercussions.

Candida albicans has historically been the most frequent cause of Candidiasis. During recent decades, several 
countries around the world have witnessed a change in the epidemiology of Candida infections, characterized by a progressive shift from a predominance of Candida albicans toward a predominance of NAC spp. (including Candida tropicalis, Candida glabrata, and Candida krusei) [1].

In order to get an insight into the present scenario of ICU in an Indian hospital, in the present prospective study, an attempt was made to isolate and speciate the Candida species from various clinical samples and to know their in vitro pattern of susceptibility to fluconazole and amphotericin $B$ and virulence patterns.

\section{Materials and Methods}

The present study was conducted from 2006 to 2008 at the Department of Microbiology, Maulana Azad Medical College and Associated Lok Nayak Hospitals, New Delhi, India, which is a 1500-bedded tertiary care hospital. One hundred and twenty-five medical/postoperative patients admitted to ICU for $>48$ hours undergoing therapy for one or more acute organ system failure or requiring intensive postoperative monitoring were studied. A total of 356 samples including blood, urine, tracheal aspirate, urinary catheter, endotracheal tube, and abdominal drains were collected and processed from the patients. Cases that had antifungal drug exposure during hospitalization were excluded from the study. All samples were inoculated on Sabouraud dextrose agar slants containing gentamicin $(0.02 \mathrm{mg} / \mathrm{mL})$ and cycloheximide $(0.5 \%)$. One set of inoculated slants was incubated at $25^{\circ} \mathrm{C}$ and the other at $37^{\circ} \mathrm{C}$, and they were examined on alternate days for the growth up to 4-6 weeks.

Identification and speciation of the isolates were conducted by colony morphology, gram staining, germ tube formation, corn meal agar with Tween80, HiCrome Candida agar, and an enzymatic triphenyltetrazolium chloride reduction; for further characterization, each isolate was subjected to carbohydrate assimilation and fermentation tests as per standard recommended procedures [7].

The in vitro minimal inhibitory concentrations (MICs) of the compounds were determined by the broth microdilution method as per Clinical and Laboratory Standards Institute (CLSI) M27-A3 document using RPMI medium and MOPS buffer. The concentration ranges tested were $0.125-128 \mu \mathrm{g} / \mathrm{mL}$ for fluconazole and $0.016-16 \mu \mathrm{g} / \mathrm{mL}$ for amphotericin B. The quality control was performed by testing C. parapsilosis (ATCC22019) and C. krusei (ATCC6258) from the American Type Culture Collection (ATCC) with each batch of clinical isolates [8]. The MIC breakpoints recommended by CLSI guidelines were followed (CLSI, 2008). For fluconazole, MIC breakpoints were as follows: sensitive, $\mathrm{MIC} \leq 8 \mu \mathrm{g} / \mathrm{mL}$; susceptible-dose dependent (SDD), MIC 16-32 $\mu \mathrm{g} / \mathrm{mL}$; resistant, $\mathrm{MIC} \geq 64 \mu \mathrm{g} / \mathrm{mL}$. For amphotericin $\mathrm{B}$, isolates with MICs of $\geq 1 \mu \mathrm{g} / \mathrm{mL}$ were categorized as resistant (CLSI, 2008).

Candida proteinase was detected by the Mohandas and Ballal method [9] using bovine serum albumin medium. The proteinase activity $(\mathrm{Pz})$ was determined in terms of the ratio of the diameter of the well to the diameter of the proteolytic zone [9]. The extracellular phospholipase activity of the isolates was screened by growing them on egg-yolk agar and measuring the size of the zone of precipitation by the Samaranayake et al. method [10]. Biofilm-formation was determined spectrophotometrically by the Branchini et al. method [11].

\section{Results}

The patients belonged to a wide age group from 13 years to 90 years with a mean age of 33 years. Out of the 125 study cases, $53(42.4 \%)$ belonged to $16-30$ years age group. Males were predominant with a male to female ratio of $1.2: 1$. In our study $86.29 \%$ patients were admitted to ICU for less than 1 week followed by $9.68 \%$ for $1-2$ weeks, while very few patients (6\%) had a stay of more than 2-3 weeks. The most common organ system involved in our patients was gastrointestinal system (27.2\% cases) followed by central nervous system (19.4\% cases) and respiratory system (19\% cases). Diabetes mellitus was seen in $14.5 \%$ cases, while fever, multiorgan system involvement, and infection were seen in $37.5 \%, 17.5 \%$, and $16 \%$ cases, respectively.

A total of 356 patient samples were collected. They included blood in 125 cases (100\%), urine in 112 cases $(89.6 \%)$, tracheal aspirate in 88 cases $(70.4 \%)$, urinary catheter in 18 cases $(14.4 \%)$, endotracheal tube in 10 cases $(8 \%)$, and abdominal drains in 3 cases (2.4\%). The most common risk factor found in our patients was that of presence of indwelling device in 100 patients followed by prolonged antibiotic therapy in 78 patients and surgery in 73 patients. Prolonged antibiotic therapy was also seen in Candidemia cases as well as Candida colonization cases (Table 1).

A total of 103 Candida isolates were obtained on culture. The majority $(39.8 \%)$ of the culture isolates were obtained from urine samples followed by tracheal aspirate (28.2\%), urinary catheter $(10.7 \%)$, blood $(9.7 \%)$, endotracheal tube (6.8\%), and abdominal drains (2.9\%). C. tropicalis $(40.8 \%)$ and C. albicans $(38.8 \%)$ were the most common Candida species causing infections in patients, followed by C. glabrata (10.7\%), C. parapsilosis (3.9\%), C. kefyr (1.9\%), C. krusei (1.9\%), and C. sphaerica (1.9\%). C. albicans (4.8\%), C. tropicalis $(3.9 \%)$ and C. glabrata $(0.9 \%)$ were the organisms incriminated in causation of candidemia (Table 2).

C. tropicalis was most often isolated from urine (35.71\%) followed by tracheal aspirate $(26.19 \%)$, urinary catheter (14.28\%), endotracheal tube (11.90\%), blood (09.52\%), abdominal drains (2.38\%), and sputum (0\%). C. albicans was more often isolated from urine $(42.50 \%)$ and tracheal aspirate (32.50\%). However, C. parapsilosis, C. krusei, C. $k e f y r$, and C. sphaerica were isolated from urine, urinary catheter, and tracheal aspirate (Table 2).

Out of the 103 Candida isolates, 6.7\% isolates showed resistance to amphotericin B, while 60 (58.25\%) showed resistance to fluconazole. The results of Table 2 showed that $67.5 \%$ C. albicans were resistant to fluconazole followed by $57.14 \%$ C. tropicalis, $50 \%$ each of C. parapsilosis and C. sphaerica, and $45.4 \%$ C. glabrata, while a lower resistance to amphotericin B of $4.7 \%, 5 \%$, and $9 \%$ was seen by C. tropicalis, C. albicans, and C. glabrata, respectively. The blood stream 
TABLE 1: Analysis of potential risk factors found for Candida infection in ICU patients.

\begin{tabular}{|c|c|c|c|c|c|}
\hline $\begin{array}{l}\text { Continuous variables } \\
(\text { mean } \pm S D)\end{array}$ & $\begin{array}{c}\text { Controls }^{\mathrm{a}} \\
(n=70,56 \%)\end{array}$ & $\begin{array}{l}\text { Candidemia } \\
(n=10,8.7 \%)\end{array}$ & $P$ value & $\begin{array}{l}\text { Candidiasis/colonization } \\
\qquad(n=45,36 \%)\end{array}$ & $P$ value \\
\hline Age (years) & $36 \pm 16.4$ & $37 \pm 17.6$ & 0.85 & $37 \pm 16.56$ & 0.75 \\
\hline $\begin{array}{l}\text { Length of hospital stay } \\
\text { (days) }\end{array}$ & $9.7 \pm 13.8$ & $7.8 \pm 4.7$ & 0.66 & $9.4 \pm 13.9$ & 0.99 \\
\hline $\begin{array}{l}\begin{array}{l}\text { Categorical variables } \\
\text { (number) }\end{array} \\
\end{array}$ & $\begin{array}{l}\text { Controls }^{\mathrm{a}} \\
(\%) n=70\end{array}$ & $\begin{array}{l}\text { Candidemia } \\
(\%) n=10\end{array}$ & $P$ value & $\begin{array}{l}\text { Candidiasis/colonization } \\
(\%) n=45\end{array}$ & $P$ value \\
\hline Surgery (73) & $42(60 \%)$ & $6(60 \%)$ & 1 & $27(67.5 \%)$ & 1 \\
\hline Trauma (28) & $18(11.4 \%)$ & $2(20 \%)$ & 0.69 & $9(20 \%)$ & 0.63 \\
\hline Pneumonia (17) & $08(11.4 \%)$ & $3(30 \%)$ & 0.2 & $9(20 \%)$ & 0.32 \\
\hline Neurological (12) & $08(11.4 \%)$ & $0(0)$ & NA & $4(8.9 \%)$ & 0.90 \\
\hline Malignancy (9) & $5(7.14 \%)$ & $1(10 \%)$ & 0.5 & $4(8.9 \%)$ & 0.73 \\
\hline TB (10) & $3(4.28 \%)$ & $0(0)$ & NA & $5(11.1 \%)$ & 0.25 \\
\hline Renal failure (3) & $0(0)$ & $0(0)$ & NA & $3(6.67 \%)$ & NA \\
\hline Heart disease (2) & $1(1.42 \%)$ & $0(0)$ & NA & $1(2.2 \%)$ & 1 \\
\hline Anemia (1) & $1(1.42 \%)$ & $0(0)$ & NA & $0(0)$ & NA \\
\hline Burns (1) & $1(1.42 \%)$ & $0(0)$ & NA & $0(0)$ & NA \\
\hline Peritonitis (23) & $09(12.85 \%)$ & $1(10 \%)$ & 1 & $12(26.67 \%)$ & 0.019 \\
\hline $\begin{array}{l}\text { Multiple organ (18) system } \\
\text { involvement }\end{array}$ & $3(4.28 \%)$ & $1(10 \%)$ & 0.4 & $15(33.3 \%)$ & 0.001 \\
\hline Indwelling devices (100) & $35(50 \%)$ & $10(100 \%)$ & NA & $45(100 \%)$ & NA \\
\hline $\begin{array}{l}\text { Prolonged antibiotic } \\
\text { therapy ( } 78)\end{array}$ & $40(57.14 \%)$ & $8(80 \%)$ & 0.3 & $33(73 \%)$ & 0.08 \\
\hline $\begin{array}{l}\text { Duration of stay }>1 \text { week in } \\
\text { ICU (34) }\end{array}$ & $11(15.71 \%)$ & $3(30 \%)$ & 0.5 & $21(46.6 \%)$ & 0.005 \\
\hline $\begin{array}{l}\text { Use of } \\
\text { immunosuppressants (32) }\end{array}$ & $13(18.57 \%)$ & $2(20 \%)$ & 0.9 & $18(40 \%)$ & 0.020 \\
\hline Diabetes mellitus (25) & $10(14.28 \%)$ & $4(40 \%)$ & 0.11 & $14(31.1 \%)$ & 0.05 \\
\hline Smoking (15) & $9(12.85 \%)$ & $2(20 \%)$ & 0.9 & $6(13.3 \%)$ & 0.9 \\
\hline
\end{tabular}

Controls $s^{\mathrm{a}}$ : patients admitted to the same ICU with no Candidemia.

Significant $P$ values $(P \leq 0.05)$ are indicated in bold. NA: not applicable.

TABLe 2: Profile of Candida species isolated from different clinical samples.

\begin{tabular}{|c|c|c|c|c|c|c|c|c|}
\hline Sample & $\begin{array}{l}\text { C. albicans } \\
\text { no. }(\%)\end{array}$ & $\begin{array}{c}\text { C. tropicalis } \\
\text { no. }(\%)\end{array}$ & $\begin{array}{c}\text { C. krusei } \\
\text { no. }(\%)\end{array}$ & $\begin{array}{c}\text { C. parapsilosis } \\
\text { no. }(\%)\end{array}$ & $\begin{array}{c}\text { C. kefyr } \\
\text { no. }(\%)\end{array}$ & $\begin{array}{c}\text { C. sphaerica } \\
\text { no. }(\%)\end{array}$ & $\begin{array}{l}\text { C. glabrata } \\
\text { no. }(\%)\end{array}$ & $\begin{array}{c}\text { Total } \\
\text { no. }(\%)\end{array}$ \\
\hline Blood & $05(4.8 \%)$ & $04(3.9 \%)$ & 0 & 0 & 0 & 0 & $01(0.9 \%)$ & $10(9.7 \%)$ \\
\hline Urine & $17(16.5 \%)$ & $15(14.6 \%)$ & $1(0.9 \%)$ & $2(1.9 \%)$ & $1(0.9 \%)$ & $1(0.9 \%)$ & $4(3.9 \%)$ & $41(39.8 \%)$ \\
\hline Sputum & 0 & 0 & 0 & 0 & 0 & 0 & $2(1.9 \%)$ & $2(1.9 \%)$ \\
\hline Urinary catheter & $3(2.9 \%)$ & $6(5.8 \%)$ & 0 & $1(0.9 \%)$ & 0 & $1(0.9 \%)$ & 0 & $11(10.7 \%)$ \\
\hline $\begin{array}{l}\text { Endotracheal } \\
\text { tube }\end{array}$ & $1(0.9 \%)$ & $5(4.8 \%)$ & 0 & 0 & 0 & 0 & $1(0.9 \%)$ & $7(6.8 \%)$ \\
\hline Tracheal aspirate & $13(12.6 \%)$ & $11(10.7 \%)$ & $1(0.9 \%)$ & 0 & $1(0.9 \%)$ & 0 & $3(2.9 \%)$ & $29(28.2 \%)$ \\
\hline $\begin{array}{l}\text { Abdominal } \\
\text { drains }\end{array}$ & $1(0.9 \%)$ & $1(0.9 \%)$ & 0 & $1(0.9 \%)$ & 0 & 0 & 0 & $3(2.9 \%)$ \\
\hline Total & $40(38.8 \%)$ & $42(40.8 \%)$ & $2(1.9 \%)$ & $4(3.9 \%)$ & $2(1.9 \%)$ & $2(1.9 \%)$ & $11(10.7 \%)$ & $103(100 \%)$ \\
\hline
\end{tabular}

isolates of C. albicans and C. tropicalis showed a resistance of $40 \%$ and $25 \%$, respectively, to fluconazole in contrast to the urinary and the indwelling isolates which were much more resistant (Table 4).

Phospholipase activity was detected in $73.78 \%$ isolates. Out of $73.78 \%$ phospholipase positive isolates, $71.05 \%$ isolates were NAC spp. and $28.95 \%$ were C. albicans. Among NAC spp., C. tropicalis $(64.81 \%)$ was the highest phospholipase producer followed by C. glabrata (20.37\%), whereas C. kefyr $(1.85 \%)$ was least producer. The proteinase activity was seen in $55.33 \%$ Candida isolates. Out of $55.33 \%$ proteinase positive isolates, $57.89 \%$ were NAC spp. and $42.10 \%$ were C. albicans. 
TABLE 3: The in vitro antifungal susceptibilities of Candida isolates to fluconazole and amphotericin B.

\begin{tabular}{|c|c|c|c|c|c|}
\hline \multirow{3}{*}{$\begin{array}{l}\text { Species } \\
\text { (no. of isolates) }\end{array}$} & \multicolumn{5}{|c|}{ Antifungal drug susceptibility pattern } \\
\hline & \multicolumn{3}{|c|}{ Fluconazole } & \multicolumn{2}{|c|}{ Amphotericin B } \\
\hline & $\begin{array}{c}S^{\mathrm{a}}(\leq 8 \mu \mathrm{g} / \mathrm{mL}) \\
\text { no. }(\%)\end{array}$ & $\begin{array}{c}\mathrm{SDD}^{\mathrm{b}}(16-32 \mu \mathrm{g} / \mathrm{mL}) \\
\text { no. }(\%)\end{array}$ & $\begin{array}{c}\mathrm{R}^{\mathrm{c}}(\geq 64 \mu \mathrm{g} / \mathrm{mL}) \\
\text { no. }(\%)\end{array}$ & $\begin{array}{l}S^{\mathrm{a}}(\leq 1 \mu \mathrm{g} / \mathrm{mL}) \\
\quad \text { no. }(\%)\end{array}$ & $\begin{array}{c}\mathrm{R}^{\mathrm{c}}(\geq 1 \mu \mathrm{g} / \mathrm{mL}) \\
\text { no. }(\%)\end{array}$ \\
\hline C. albicans (40) & $10(25 \%)$ & $3(7.5 \%)$ & 27 (67.5\%) & 38 (95\%) & $2(5 \%)$ \\
\hline C. tropicalis (42) & $15(35.7 \%)$ & $3(7.1 \%)$ & $24(57.2 \%)$ & $40(95.2 \%)$ & $2(4.8 \%)$ \\
\hline C. glabrata (11) & $6(54.5 \%)$ & 0 & $5(45.5 \%)$ & $10(90.9 \%)$ & $1(9.1 \%)$ \\
\hline C. sphaerica (02) & $1(50 \%)$ & 0 & $1(50 \%)$ & $2(100 \%)$ & 0 \\
\hline C. parapsilosis (04) & $2(50 \%)$ & 0 & $2(50 \%)$ & $4(100 \%)$ & 0 \\
\hline C. kefyr (02) & $2(100 \%)$ & 0 & 0 & $2(100 \%)$ & 0 \\
\hline C. krusei (02) & 0 & $1(50 \%)$ & $1(50 \%)$ & 0 & $2(100 \%)$ \\
\hline
\end{tabular}

$S^{a}$ : susceptible range.

$\mathrm{SDD}^{\mathrm{b}}$ : susceptible dose-dependent range.

$\mathrm{R}^{\mathrm{c}}$ : resistant range.

Among NAC spp., C. tropicalis (66.66\%) was the highest proteinase producer followed by C. glabrata (12.12\%), whereas C. kefyr showed no proteinase activity. The adherence activity was shown by $99.02 \%$ Candida isolates; out of these isolates $10.78 \%$ were weak positive $\{2+\}$ and $19.60 \%$ strong positive $\{3+\}$, while $69.61 \%$ isolates showed maximum adherent activity $\{4+\}$. NAC species $(60.78 \%)$ produced biofilm in comparison to C. albicans (39.22\%) showing relatively lower slime production. Also stronge biofilm production was seen in cases of $C$. tropicalis compared to weak biofilm production that was seen in C. kefyr, C. krusei, and C. sphaerica.

\section{Discussion}

Patients in ICU are at a higher risk of acquiring nosocomial infections compared with patients in general wards due to the severity of the underlying illnesses and iatrogenic factors related to the high frequency of invasive procedures needed for the monitoring and treatment [12] which include insertion of intravascular catheters, endotracheal intubation, and positive pressure ventilation, urinary catheterization and surgical operations.

Studies from different parts of the world on nosocomial infections have shown that patient and treatment factors are risk factors for the development of nosocomial infections. Some identified risk factors related to treatment have been length of hospital or ICU stay, presence of indwelling devices, and intake of drugs like broad spectrum antibiotics and immunosuppressants [13] which were also seen in our cases as $86.29 \%$ patients were admitted to ICU for less than 1 week followed by $9.68 \%$ for $1-2$ weeks. According to Sydnor and Perl, admission to ICU itself was a significant risk factor; roughly $25 \%$ of nosocomial infections occur in intensive care units (ICUs), which have been estimated to increase ICU length of stay by 4.3 to 15.6 days [14]. Length of stay in the ICU is also associated with increased risk for Candida infections, which rises rapidly after 7-10 days [1]. History of smoking was elicited in $26 \%$ of cases corroborated by a recent study from Mumbai also showing the association of nosocomial pneumonia and mortality with tobacco smoking [15]. Workers from Delhi have reported a higher risk of nosocomial infections in ICUs attributed to the severity of the underlying illness and the development of iatrogenic factors related to the high frequency of invasive procedures required in these patients [12].

Peritonitis, multiple organ system involvement, duration of stay $>1$ week, and immunosuppressant were significantly associated with the group with Candida colonization $(P \leq$ 0.05) unlike the Candidemia cases. The number of cases of Candidemia was may be too low to get a significant association. Similar association with antibiotic and immunosuppressant therapy along with catheterization and surgery in causation of nosocomial Candidiasis has been quoted in other studies in North India $[16,17]$.

In ICU patients, the most common types of Candida infections are seen to comprise bloodstream, catheter-related, intra-abdominal, and urinary tract infections [6]. In our patients, culture isolations were obtained in $39.8 \%$ of urine samples followed by $31.06 \%$ of tracheal aspirate in addition to a varying percentage from other samples like urinary catheter (10.6\%), blood (8.7\%), endotracheal tube (6.7\%), and abdominal drains samples (2.9\%). Different studies reported the predominance of $C$. albicans as a leading cause of invasive candidiasis. However, recently, several countries around the world have witnessed a change in the epidemiology of Candida infections, characterized by a progressive shift from a predominance of $C$. albicans toward a predominance of NAC species [1]. Although C. albicans remains the most commonly isolated species from cases of Candidemia in USA, Europe, and South America (Brazil), its dominance is slowly giving way to an increase in NAC species such as C. glabrata [18].

However, in India, C. tropicalis appears to be emerging as the most common cause of Candidemia [18]. $50 \%$ of our cases of Candidemia were due to C. albicans followed by C. tropicalis (40\%) and C. glabrata (10\%). Findings of our study quite similar to the epidemiological data from the Indian subcontinent showed that $50 \%$ of our nosocomial candidemia cases were due to NAC spp. of which C. tropicalis was the most dominant [19]. 


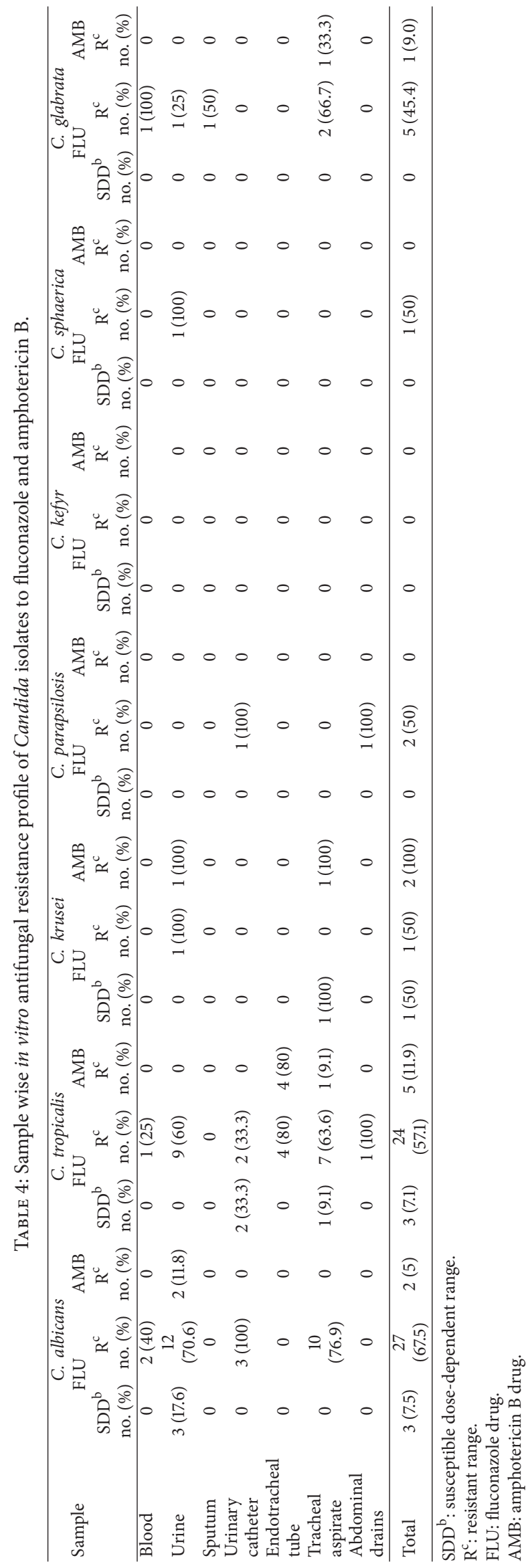


Interestingly, in our study, C. tropicalis was most often isolated from urine $(35.71 \%)$ followed by tracheal aspirate $(26.19 \%)$, urinary catheter $(14.28 \%)$, endotracheal tube (11.90\%), blood (09.52\%), abdominal drains (2.38\%), and sputum (0\%). C. albicans was more often isolated from urine $(42.50 \%)$ and tracheal aspirate (32.50\%). However, $C$. parapsilosis, C. krusei, C. kefyr, and C. sphaerica were isolated only from urine, urinary catheter, and tracheal aspirate. $36.9 \%$ of isolates of $C$. tropicalis were from colonization sites (Table 2).

Some of these emerging species have been correlated with increased virulence. An increasing role for NAC spp. was also noticed in studies performed among ICU patients, although the issue is somewhat controversial [20]. The increased use of fluconazole has been seen to be associated with the predominance of many NAC spp., especially $C$. tropicalis species[21]. Findings in our study have confirmed and corroborated the earlier reports of increased resistance to fluconazole among isolates of $C$. tropicalis from the AsiaPacific region [22]. Also C. parapsilosis, C. krusei, C. kefyr, and C. sphaerica have shown a predilection towards isolation from urinary catheters and tracheal aspirates in our cases, explaining their role in inherent biofilm formation leading to difficulty in eradication from the site and development of resistance in their long stay. Several investigators have postulated that the widespread use of fluconazole would have a big role in selecting out yeast species intrinsically resistant or less sensitive to fluconazole, such as C. krusei, C. glabrata, or C. tropicalis [20]. In our study, a large proportion of the C. tropicalis $(57.14 \%, 24 / 42)$ was resistant to fluconazole; 90.4\% of C. tropicalis isolates were responsible for Candida colonization with many of them (50\%) being involved in biofilm formation. Punithavathy et al. [23] from South India, however, reported that $(22 \%, 11 / 50)$ isolates of $C$. tropicalis were resistant to fluconazole.

Blood stream isolates of C. albicans, C. tropicalis were $40 \%$ and $25 \%$ while the urinary isolates were $70.6 \%$ and $60 \%$ resistant to fluconazole respectively, in comparison to a higher resistance going up to $100 \%$ seen in isolates of endotracheal tubes, tracheal aspirate and abdominal drains. Our results of $25 \%$ resistance to fluconazole in blood stream isolates of $C$. tropicalis were consistent with a report from Maharashtra and Delhi, in which $37 \%$ and up to $83 \%$, respectively, were resistant to fluconazole [24, 25], while the isolates from urine and indwelling devices showed a higher resistance quite like a similar picture being seen in Chennai, South India, reflecting the high level of resistance in colonized isolates involved in biofilm formation predisposing to candidemia [26].

Candida glabrata has also become important because of its increasing incidence worldwide; it was seen in $11.6 \%$ of our study cases with 10 out 11 isolates from colonization sites exhibiting a high resistance to azoles. C. krusei, found in 2 cases again from colonization sites in our study, is another important Candida species which is gaining clinical significance because of its intrinsic resistance toward azoles and poor susceptibility to all other antifungals, including amphotericin B [27]. C. sphaerica was isolated from urine and urinary catheter samples only and $50 \%$ of C. sphaerica isolates were resistant to fluconazole, while no resistance to amphotericin B was seen. On the other hand both the isolates of $C$. kefyr from urine and tracheal aspirate were sensitive to fluconazole and amphotericin B. However, in a study in Turkey, all the C. sphaerica strains (3/3) were sensitive to Fluconazole and resistant to amphotericin B and 25\% (2/8) Candida kefyr strains were susceptible dose dependent (SDD) and $37.5 \%(3 / 8)$ sensitive to fluconazole and $37.5 \%$ (3/8) were SDD and 25\% (2/8) resistant to amphotericin B as reported by Abaci and Haliki-Uztan [28] (Table 3).

Virulence activity of C. albicans and C. tropicalis isolates from blood samples were $40 \%$ and $75 \%$ for phospholipaseactivity, $60 \%$ and $25 \%$ for proteinase-activity and $100 \%$ each for adherent-activity, respectively. But the same was much more in the biofilm isolates from urinary catheter, endotracheal tube, and tracheal aspirate: $33.3 \%$ to $100 \%$ for phospholipase activity, $61.5 \%$ to $100 \%$ for proteinase activity, and $100 \%$ adherent activity in the urinary catheter, endotracheal tube and tracheal aspirate going up to $100 \%$ phospholipase activity, proteinase activity, and adherent activity in abdominal drains.

The proteinase-producing capacity of $C$. albicans (42.10\%) was less than that of NAC spp. (57.89\%) in our patients, whereas workers from Manipal showed NAC spp. $(50.45 \%)$ to be lower proteinase producers than C. albicans $(67.34 \%)$ [29]. In our study among NAC spp. C. tropicalis $(66.66 \%)$ was the highest proteinase producer, whereas $C$. kefyr showed no proteinase activity consistent with studies of Al-Nedaithy [30] which showed C. tropicalis (95\%) to be proteinase producer and no activity with C. kefyr. 50-100\% of our Candida isolates showed phospholipase activity similar to the previous studies conducted by Price et al. [31] and $\mathrm{Wu}$ et al. [32] who reported phospholipase activity in 30-100\% of their Candida isolates from various groups of patients and from various sites. Also a total of $22(28.95 \%)$ of C. albicans isolates and $54(85.7 \%)$ of NAC spp. isolates produced phospholipase in our cases, while Mohandas and Ballal [29] from Manipal and Ibrahim et al. [33] reported 23 (46.93\%) of their C. albicans isolates and 26 (42\%) of NAC spp. isolates producing phospholipase with greater extracellular phospholipase activity. In our study among NAC spp., $C$. tropicalis $(64.81 \%)$ was the highest phospholipase producer concordant with studies of Negri et al. [34] and Júnior Jr. et al. [35] showing C. tropicalis to be the species with the highest number of positive isolates for phospholipase activity (up to $91.7 \%$ ).

Biofilm production has been seen to promote persistence of infection as was evidenced in Candida isolates of OPC patient in a major medical center in India [36]. Our study showed that $60.78 \%$ of isolates of NAC spp. produced biofilm in comparison to $39.21 \%$ of C. albicans producing lower slime (Figure 1). Shin et al. [5] and Pathak et al. [37] also reported higher biofilm-forming ability of NAC spp. than C. albicans species. Our data also suggest the production of stronger biofilms by $C$. tropicalis $(40.19 \%)$ followed by $C$. albicans $(39.21 \%)$. Weak biofilm production was seen with C. kefyr, C. krusei, and C. sphaerica (1.96\% each). Biofilm positivity has also been seen to occur most frequently in C. tropicalis isolates followed by C. parapsilosis, C. glabrata, 


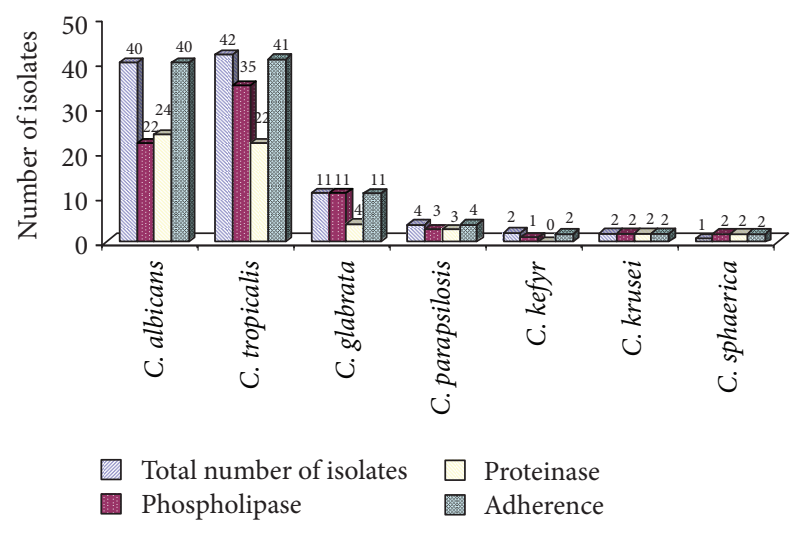

FIGURE 1: Pathogenicity profile of Candida species isolates; NAC spp. had greater biofilm forming ability in comparison to Candida albicans species.

and C. albicans [5]. Kumar et al. [38] also reported $C$. tropicalis to be strongly positive for biofilm production. However, Hawser and Douglas [39] reported that isolates of C. parapsilosis and C. glabrata were significantly less likely to produce biofilms than the more pathogenic C. albicans. Biofilm may be a specific trait of a Candida that is not associated with differences in adherence but with slowness of growth; however, Candida biofilms contribute both to the pathogenesis of superficial and systemic Candidiasis as they are notoriously resistant to antifungal drugs [36].

Advances in modern medicine and patient management have seen increased use of prosthetic biomaterials. This has led to the complication associated with biofilm formation on these materials, the constituent organisms of which are different in their growth rate and antifungal susceptibility as compared to isolates not associated with biofilms. The knowledge of the local epidemiological trends in the Candida species isolated is important to guide therapeutic choices. Our study demonstrates a shift of C. albicans predominance towards a NAC spp. in causation of candidiasis among ICU population with the isolation of $C$. tropicalis from a large number of cases highlighting the importance of this pathogen. The presence of high resistance to fluconazole by $C$. albicans followed by $C$. tropicalis is another important finding in our study and may have important clinical repercussions. It was seen that NAC spp. had greater biofilm-forming ability when compared to C. albicans species especially $C$. tropicalis showed the highest ability to produce phospholipase, proteinase, and biofilms. Thus in the light of virtual absence of such data from our area, this study would help in understanding the properties of the biofilms, with respect to growth characteristics and drug sensitivity, and should be a great help in understanding the present trends and predicting the overall long-term trends that will be helpful to physicians and to antibiotic use committees in establishing guidelines for the appropriate use of antifungal agents. This will help in reducing complications associated with biofilm formation and devise control strategies and consequently reducing morbidity, mortality, and treatment costs. The results obtained here open perspectives of new investigations that are aimed at establishing and broadening knowledge of understanding relationship between strain types and properties such as pathogenicity, commensality, and infectivity, an important interplay in host-pathogen relationship specially as far as Candida infections are concerned.

\section{Conflict of Interests}

The authors declare that they have no conflict of interests.

\section{References}

[1] M. Méan, O. Marchetti, and T. Calandra, "Bench-to-bedside review: Candida infections in the intensive care unit," Critical Care, vol. 12, no. 1, article 204, 2008.

[2] J. E. Edwards Jr., "Invasive Candida infections: evolution of a fungal pathogen," The New England Journal of Medicine, vol. 324, no. 15, pp. 1060-1062, 1991.

[3] C. M. Beck-Sague and W. R. Jarvis, "Secular trends in the epidemiology of nosocomial fungal infections in the United States, 1980-1990," Journal of Infectious Diseases, vol. 167, no. 5, pp. 1247-1251, 1993.

[4] V. Mohandas and M. Ballal, "Biofilm as virulence marker in Candida isolated from blood," World Journal of Medical Sciences, vol. 2, no. 1, pp. 46-48, 2007.

[5] J. H. Shin, S. J. Kee, M. G. Shin et al., "Biofilm production by isolates of Candida species recovered from nonneutropenic patients: comparison of bloodstream isolates with isolates from other sources," Journal of Clinical Microbiology, vol. 40, no. 4, pp. 1244-1248, 2002.

[6] P. Eggimann, J. Garbino, and D. Pittet, "Management of Candida species infections in critically ill patients," The Lancet Infectious Diseases, vol. 3, no. 12, pp. 772-785, 2003.

[7] E. W. Koneman, S. D. Allen, W. M. Janda et al., "Mycology," in Color Atlas and Textbook of Diagnostic Microbiology, pp. 9831057, Lippincott Williams and Wilkins, Philadelphia, Pa, USA, 5th edition, 1997.

[8] Clinical and Laboratory Standards Institute, "Reference method for broth dilution antifungal susceptibility testing of yeasts, Approved Standard-third edition," CLSI Document M27-A3, Clinical and Laboratory Standards Institute (CLSI), 2008.

[9] V. Mohandas and M. Ballal, "Proteinase and phospholipase activity as virulence factors in Candida species isolated from blood," Revista Iberoamericana de Micologia, vol. 25, no. 4, pp. 208-210, 2008.

[10] Y. H. Samaranayake, R. S. Dassanayake, J. A. M. S. Jayatilake et al., "Phospholipase B enzyme expression is not associated with other virulence attributes in Candida albicans isolates from patients with human immunodeficiency virus infection," Journal of Medical Microbiology, vol. 54, no. 6, pp. 583-593, 2005.

[11] M. L. Branchini, M. A. Pfaller, J. Rhine-Chalberg, T. Frempong, and H. D. Isenberg, "Genotypic variation and slime production among blood and catheter isolates of Candida parapsilosis," Journal of Clinical Microbiology, vol. 32, no. 2, pp. 452-456, 1994.

[12] G. P. Dureja, "Nosocomial Infections in Intensive Care Unit: a study," Hospital Today, vol. 11, pp. 695-699, 2001.

[13] J. Freeman and J. E. McGonan Jr., "Methodological issues in hospital epidemiology: rates, core finding and interpretation," Reviews of Infectious Diseases, vol. 3, pp. 668-677, 1981. 
[14] E. R. M. Sydnor and T. M. Perl, "Hospital epidemiology and infection control in acute-care settings," Clinical Microbiology Reviews, vol. 24, no. 1, pp. 141-173, 2011.

[15] P. H. Chandrasekar, J. A. Kruse, and M. F. Mathews, "Nosocomial infection among patients in different types of intensive care units at a city hospital," Critical Care Medicine, vol. 14, no. 5, pp. 508-510, 1986.

[16] A. Chakrabarti, K. Singh, and S. Das, "Changing face of nosocomial Candidemia," Indian Journal of Medical Microbiology, vol. 17, pp. 160-166, 1999.

[17] I. Xess, N. Jain, F. Hasan, P. Mandal, and U. Banerjee, "Epidemiology of candidemia in a tertiary care centre of North India: 5year study," Infection, vol. 35, no. 4, pp. 256-259, 2007.

[18] R. I. Singh, I. Xess, P. Mathur, B. Behera, B. Gupta, and M. C. Misra, "Epidemiology of candidaemia in critically ill trauma patients: experiences of a level I trauma centre in North India," Journal of Medical Microbiology, vol. 60, no. 3, pp. 342-348, 2011.

[19] R. J. Kothavade, M. M. Kura, A. G. Valand, and M. H. Panthaki, "Candida tropicalis: its prevalence, pathogenicity and increasing resistance to fluconazole," Journal of Medical Microbiology, vol. 59, no. 8, pp. 873-880, 2010.

[20] M. Bassetti, E. Righi, A. Costa et al., "Epidemiological trends in nosocomial candidemia in intensive care," BMC Infectious Diseases, vol. 6, article 21, 2006.

[21] S. Giri and A. J. Kindo, "A review of Candida species causing blood stream infection," International Journal of Medical Microbiology, vol. 30, no. 3, pp. 270-278, 2012.

[22] M. A. Pfaller, D. J. Diekema, D. L. Gibbs et al., "Results from the artemis disk global antifungal surveillance study, 1997 to 2007: a 10.5-year analysis of susceptibilities of candida species to fluconazole and voriconazole as determined by CLSI standardized disk diffusion," Journal of Clinical Microbiology, vol. 48, no. 4, pp. 1366-1377, 2010.

[23] P. M. Punithavathy, K. Nalina, and T. Menon, "Antifungal susceptibility testing of Candida tropicalis biofilms against fluconazole using calorimetric indicator resazurin," Indian Journal of Pathology and Microbiology, vol. 55, no. 1, pp. 72-74, 2012.

[24] S. C. Deorukhkar and S. Saini, "Species distribution and antifungal susceptibility profile of Candida species isolated from blood stream infections," Journal of Evolution of Medical and Dental Sciences, vol. 1, no. 3, pp. 241-249, 2012.

[25] A. Kothari and V. Sagar, "Epidemiology of Candida bloodstream infections in a tertiary care institute in India," Indian Journal of Medical Microbiology, vol. 27, no. 2, pp. 171-172, 2009.

[26] C. P. G. Kumar, T. Sundararajan, T. Menon, and M. Venkatadesikalu, "Candidosis in children with onco-hematological diseases in Chennai, South India," Japanese Journal of Infectious Diseases, vol. 58, no. 4, pp. 218-221, 2005.

[27] J. F. Ernst and A. Schmidt, Dimorphism in Human Pathogenic and A Pathogenic Yeasts, S. Karger AG, Basel, Switzerland, 2000.

[28] O. Abaci and A. Haliki-Uztan, "Investigation of the susceptibility of Candida species isolated from denture wearers to different antifungal antibiotics," African Journal of Microbiology Research, vol. 5, no. 12, pp. 1398-1403, 2011.

[29] V. Mohandas and M. Ballal, "Distribution of Candida Species in different clinical samples and their virulence: biofilm formation, proteinase and phospholipase production: a study on hospitalized patients in Southern India," Journal of Global Infectious Diseases, vol. 3, no. 1, pp. 4-8, 2011.

[30] S. S. A. Al-Nedaithy, "Spectrum and proteinase production of yeasts causing vaginitis in Saudi Arabian women," Medical Science Monitor, vol. 8, no. 7, pp. CR498-CR501, 2002.
[31] M. F. Price, I. D. Wilkinson, and L. O. Gentry, "Plate method for detection of phospholipase activity in Candida albicans," Sabouraudia Journal of Medical and Veterinary Mycology, vol. 20, no. 1, pp. 7-14, 1982.

[32] T. Wu, L. P. Samaranayake, B. Y. Cao, and J. Wang, "In-vitro proteinase production by oral Candida albicans isolates from individuals with and without HIV infection and its attenuation by antimycotic agents," Journal of Medical Microbiology, vol. 44, no. 4, pp. 311-316, 1996.

[33] A. S. Ibrahim, F. Mirbod, S. G. Filler et al., "Evidence implicating phospholipase as a virulence factor of Candida albicans," Infection and Immunity, vol. 63, no. 5, pp. 1993-1998, 1995.

[34] M. Negri, M. Martins, M. Henriques, T. I. E. Svidzinski, J. Azeredo, and R. Oliveira, "Examination of potential virulence factors of Candida tropicalis clinical isolates from hospitalized patients," Mycopathologia, vol. 169, no. 3, pp. 175-182, 2010.

[35] A. D. Júnior Jr., A. F. Silva, F. C. Rosa, S. G. Monteiro, P. de Maria Silva Figueiredo, and C. de Andrade Monteiro, "In vitro diferential activity of phospholipases and acid proteinases of clinical isolates of Candida," Revista da Sociedade Brasileira de Medicina Tropical, vol. 44, no. 3, pp. 334-338, 2011.

[36] F. Hasan, I. Xess, X. Wang, N. Jain, and B. C. Fries, "Biofilm formation in clinical Candida isolates and its association with virulence," Microbes and Infection, vol. 11, no. 8-9, pp. 753-761, 2009.

[37] A. K. Pathak, S. Sharma, and P. Shrivastva, "Multi-species biofilm of Candida albicans and non-Candida albicans Candida species on acrylic substrate," Journal of Applied Oral Science, vol. 20, no. 1, pp. 70-75, 2012.

[38] A. Kumar, V. C. Thakur, S. Thakur et al., "Phenotypic characterization and in vitro examination of potential virulence factors of Candida species isolated from blood stream infection," World Journal of Science and Technology, vol. 1, no. 10, pp. 38-42, 2011.

[39] S. P. Hawser and L. J. Douglas, "Biofilm formation by Candida species on the surface of catheter materials in vitro," Infection and Immunity, vol. 62, no. 3, pp. 915-921, 1994. 

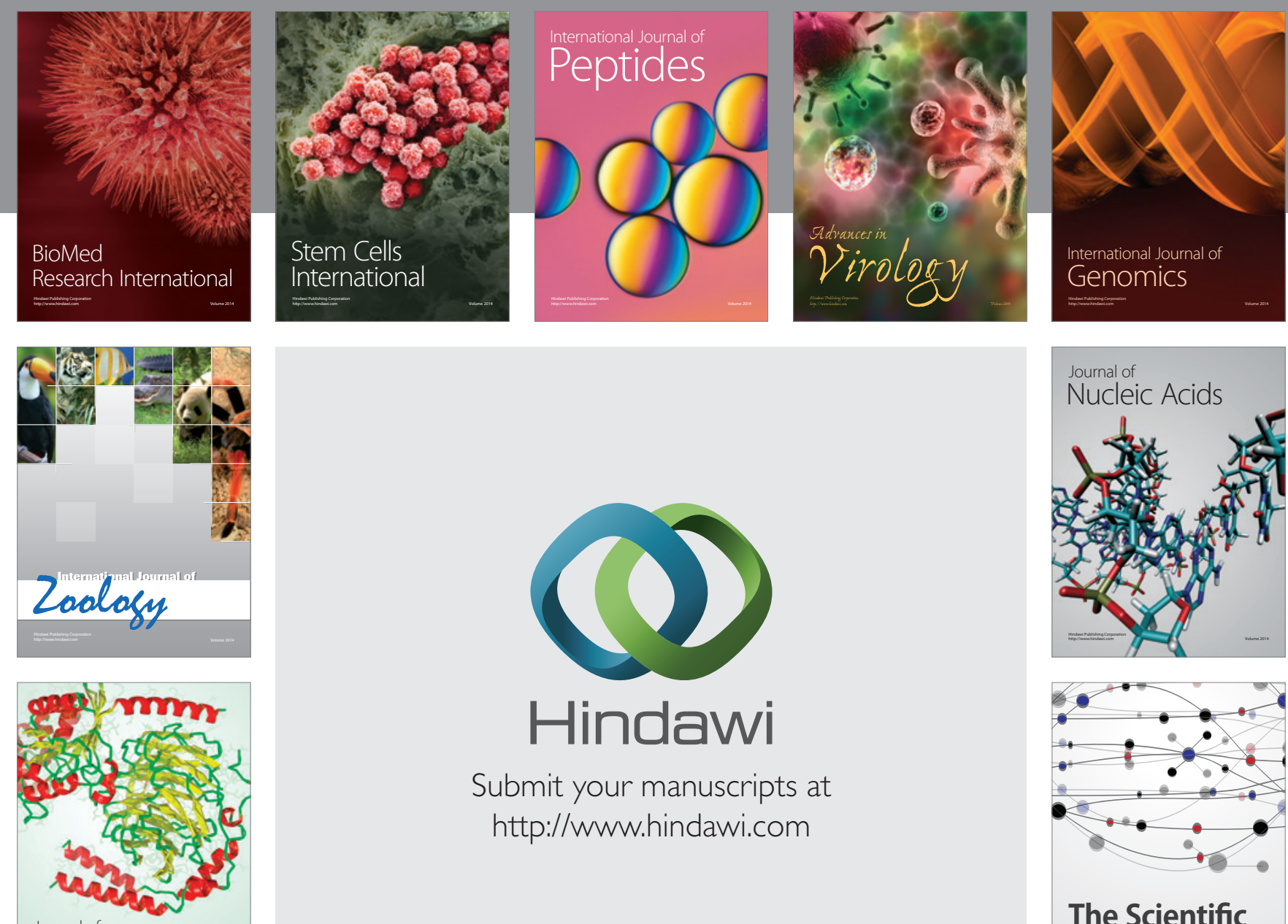

Submit your manuscripts at

http://www.hindawi.com

Journal of
Signal Transduction
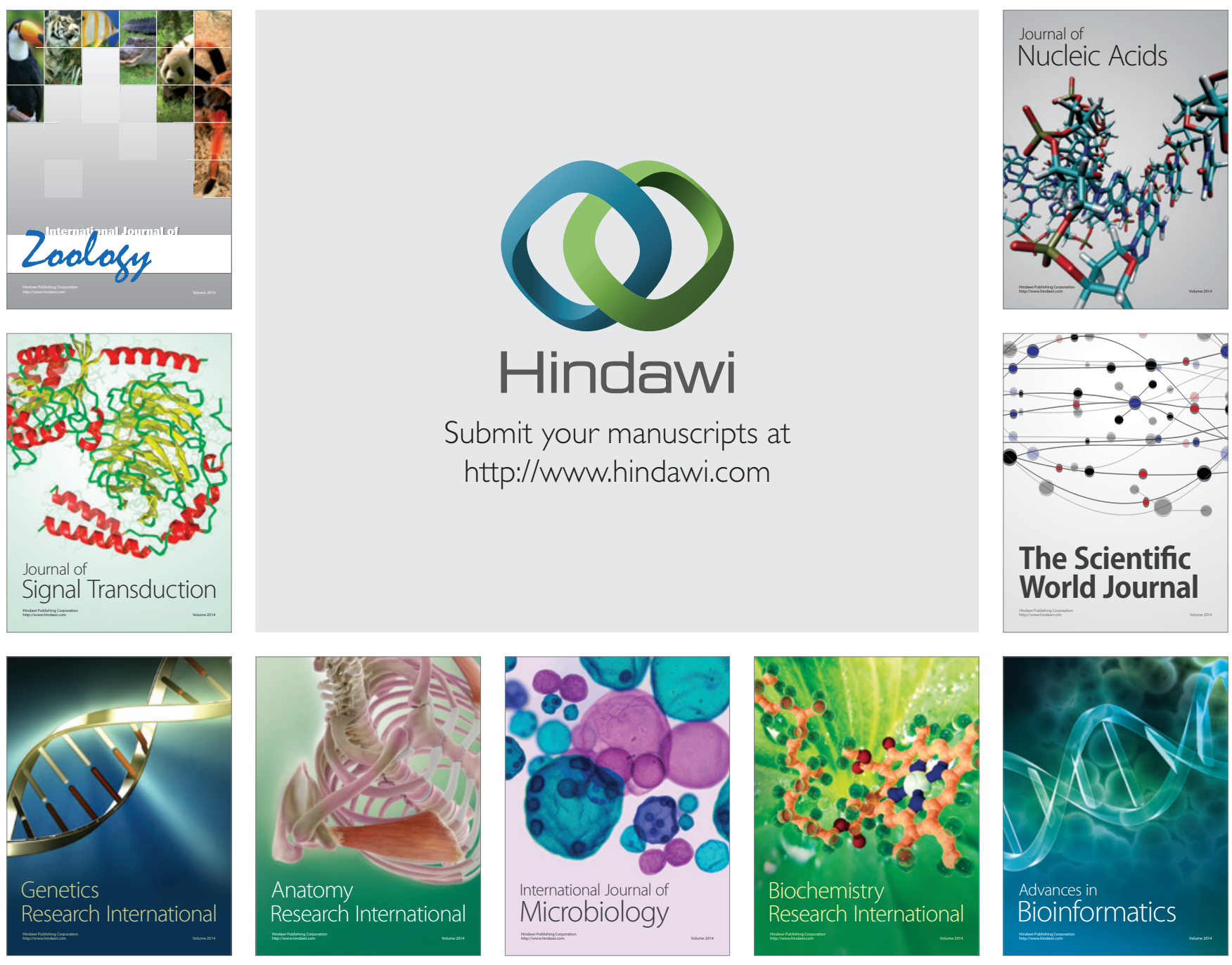

The Scientific World Journal
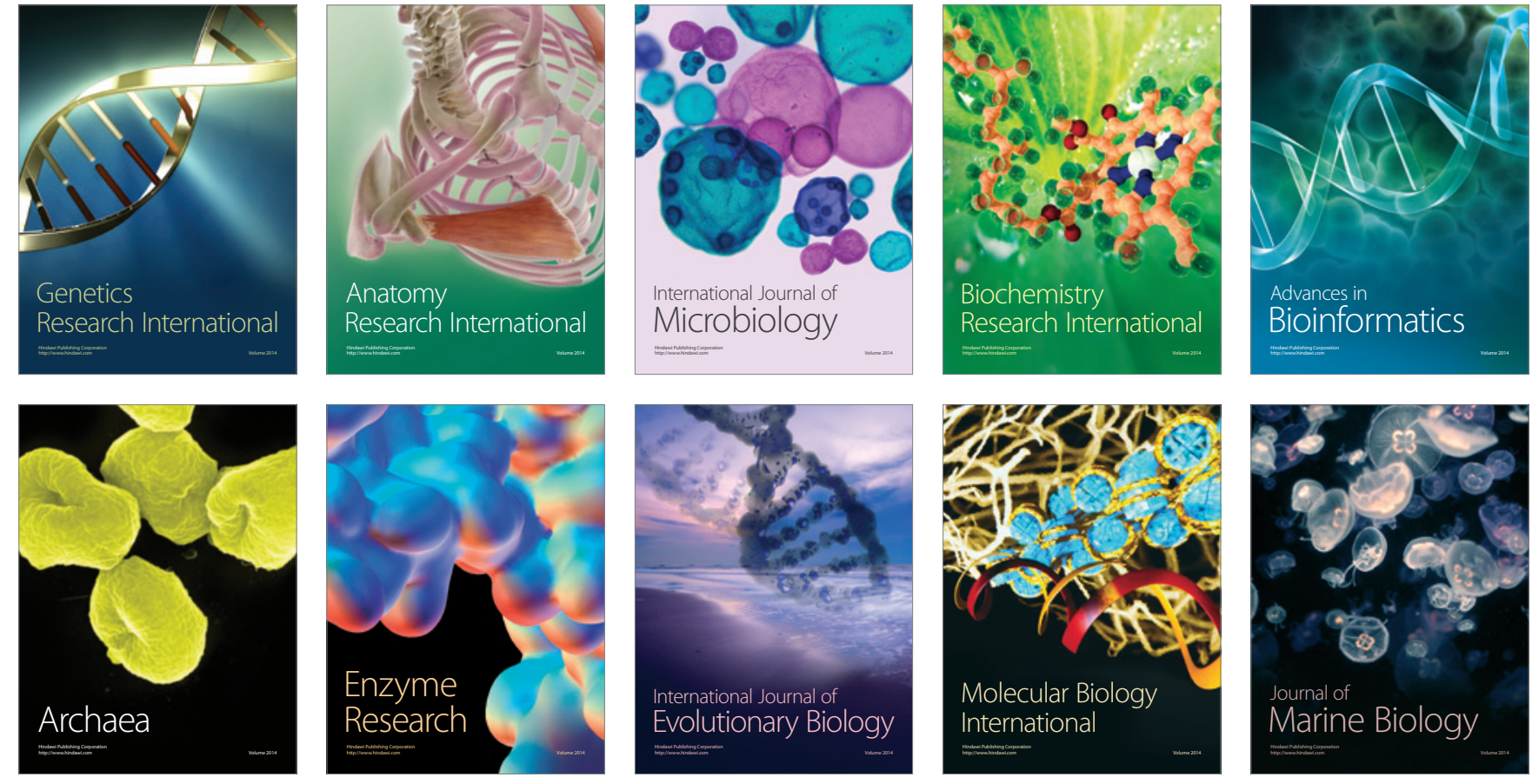